\title{
Manipulating the gut microbiota to combat alcoholic hepatitis
}
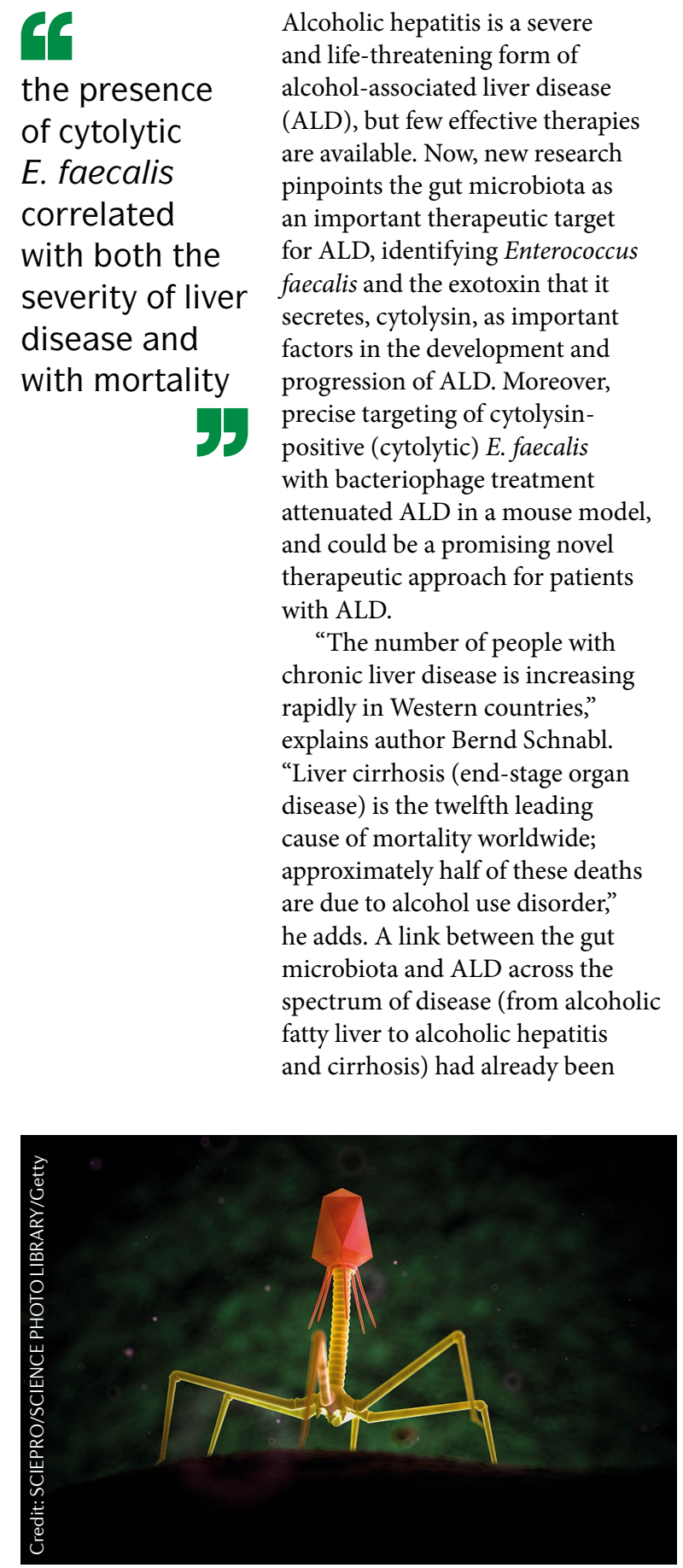

demonstrated, but the underlying mechanisms and precise role of microorganisms were unknown. Previous work had shown that ALD could be transferred via faecal microbiota transplantation from patients with alcoholic hepatitis in germ-free mice, and so Schnabl and colleagues wanted to investigate the bacteria and toxins responsible for this transmissible phenotype and their role in ALD development.

First, the investigators examined the gut microbiota in different patient cohorts, and confirmed that chronic alcohol use $(n=44)$ and alcoholic hepatitis $(n=88)$ were associated with alterations in the composition of the faecal microbiota compared with individuals without alcohol-use disorders as controls $(n=26)$. One notable difference was the increased proportion of Enterococcus spp. in patients with alcoholic hepatitis (accounting for $5.59 \%$ of faecal bacteria versus almost none identified in faecal bacteria from controls). Moreover, faecal samples from patients with alcoholic hepatitis had $~ 2,700$-fold more E. faecalis than controls, with $\sim 80 \%$ of patients with alcoholic hepatitis positive for E. faecalis in faeces. Importantly, the presence of cytolytic E. faecalis correlated with both the severity of liver disease and with mortality in these patients; most cytolysinpositive patients with alcoholic hepatitis (89\%) died within 180 days of admission.

Next, the researchers used mouse models of ALD to demonstrate that cytolysin directly promoted disease development. Importantly, cytolysin directly induced hepatocyte death in vitro, and promoted liver injury in the context of ethanol-induced liver disease (chronic-binge ethanol diet) in mice. Mice colonized with cytolytic E. faecalis and then fed ethanol had more severe liver injury and increased hepatic steatosis than controls. The same trend in ALD severity was observed in germ-free mice that received faecal transplants from cytolysin-positive patients with alcoholic hepatitis.

Finally, the researchers developed a novel therapeutic approach using bacteriophages to target cytolytic E. faecalis specifically. In a humanized mouse model of ALD, bacteriophage treatment decreased cytolysin in the liver and abolished ethanol-induced liver injury and steatosis.

"This novel bacteriophage approach might be developed to alter the intestinal microbiomes of and treat patients with alcoholic hepatitis," says Schnabl. "Phage therapy offers the opportunity to very precisely edit the gut microbiota and selectively target specific, toxin-producing bacterial strains."

The researchers call for more studies to validate their findings. "An independent cohort of patients with alcoholic hepatitis is required to validate the human relevance of our findings and to test cytolysin as a predictive biomarker," notes Schnabl, adding that a phase I clinical safety study using bacteriophage therapy in patients with alcoholic hepatitis is necessary to move forwards to the clinic.

Katrina Ray

ORIGINAL ARTICLE Duan, Y. et al. Bacteriophage targeting of gut bacterium attenuates alcoholic liver disease. Nature 575, 505-511 (2019) RELATED ARTICLE Bajaj, J. S. Alcohol, liver disease and the gut microbiota. Nat. Rev. Gastroenterol. Hepatol. 16, 235-246 (2019) 